\title{
Research Article \\ Effective Permittivity of Biological Tissue: Comparison of Theoretical Model and Experiment
}

\author{
Li Gun, Du Ning, and Zhang Liang \\ Department of Biomedical Engineering, School of Electronic Information Engineering, Xian Technological University, \\ Xian 710021, China \\ Correspondence should be addressed to Li Gun; ligun@xatu.edu.cn
}

Received 12 March 2017; Revised 14 May 2017; Accepted 25 May 2017; Published 21 June 2017

Academic Editor: Marek Lefik

Copyright (C) 2017 Li Gun et al. This is an open access article distributed under the Creative Commons Attribution License, which permits unrestricted use, distribution, and reproduction in any medium, provided the original work is properly cited.

Permittivity of biological tissue is a critical issue for studying the biological effects of electromagnetic fields. Many theories and experiments were performed to measure or explain the permittivity characteristics in biological tissue. In this paper, we investigate the permittivity parameter in biological tissues via theoretical and experimental analysis. Firstly, we analyze the permittivity characteristic in tissue by using theories on composite material. Secondly, typical biological tissues, such as blood, fat, liver, and brain, are measured by HP4275A Multi-Frequency LCR Meter within $10 \mathrm{kHz}$ to $10 \mathrm{MHz}$. Thirdly, experimental results are compared with the Bottcher-Bordewijk model, the Skipetrov equation, and the Maxwell-Gannett theory. From the theoretical perspective, blood and fat are regarded as the composition of liver and brain because of the high permittivity in blood and the opposite in fat. Volume fraction of blood in liver and brain is analyzed theoretically, and the applicability and the limitation of the models are also discussed. These results benefit further study on local biological effects of electromagnetic fields.

\section{Introduction}

Biological effects induced by electromagnetic radiation depend on electric properties of biological tissue during exposure to electromagnetic radiation. Because of difficulties in measurement of biological tissue in vivo and permittivity of biological tissue which shows nonlinear characteristics in frequency domain, determining effective permittivity of biological tissue has received repeated attention in recent years [1-3]. Many techniques and principles of testing permittivity in biological tissue are similar to the traditional engineering materials. Capacitance of the tissue is measured first, and then permittivity can be calculated by the test results via relationship between the tissue morphology and the electromagnetic theory [4-6]. That is, the experimental study on permittivity of biological tissue is conducted via calculation from the test result (capacitance). The existing research has already reflected a lot of specific application. Otaki et al. suggested that dielectric blood coagulometry may be a useful method for measuring blood clotting and could provide the detailed assessment for the status of anticoagulant therapy [7]. The dielectric properties are not stable in biological system, which changes with external conditions such as temperature and external electric frequency. The dielectric properties of living tissue have its own features, and calculation of effective permittivity based on theory modeling can greatly reduce the workload in exploring them [8]. There are already many theoretical studies on simulation of effective permittivity in biological tissue by using perturbation expansion, effective medium approximation, the finite element method, and so on [9-12]. Among the studies on mathematical models of permittivity in tissue, most of them used the composite material model. Ashutosh Prasad et al. study experimental data yielded by different mixture equations in order to test the acceptability of dielectric mixture equations for high volume fractions of the inclusion material in the mixture [13]. Amooey considers the modification of volume fraction to improve mixing rules model on calculation of effective permittivity. Some mixing rules are compared for studying the permittivity of mixtures [14]. Zohdi et al. take for electromagnetic techniques which may provide an effective way to estimate the permittivity in blood, which can determine the volume fraction of blood cell in other tissue. They also attend to think that the deviation of the permittivity parameter 
can help characterize certain tissue disorders [15]. Prodan develops a theoretical framework for describing the dielectric response of live cells under low external electric fields by taking the presence of the cell's membrane and its charge movement into account, and then the author also analyzed the effect of several other physical parameters on permittivity in cell [16]. Peón-Fernández et al. established a numerical model to estimate permittivity for periodically structured materials via the long wave approximation. When the system was subjected to a prescribed potential, Monte-Carlo random walk iterative method was used to estimate the numerical result, and the model can well evaluate field distribution. They also suggested that values of the effective permittivity for certain aggregate systems such as percolating simple cubic structures are important for the usage of biological tissue [17].

From the contents of the above-mentioned literatures, there is no study combining the experimental results and theory. This paper deals with the effective permittivity in biological system, especially in the blood, liver, fat, and brain. A theoretical analysis on several typical model of effective permittivity in materials is presented. We intend to explore the acceptability of the models in biotissues. So we take for the well feasibility of the volume fractions model such as the Bottcher-Bordewijk model, the Skipetrov equation, and the Maxwell-Wagner theory to analyze the acceptability of these models. Finally, experiment is conducted to validate the acceptability of the model mentioned.

\section{Theoretical Models and Their Applicability}

The permittivity in biological system is related to its structure, compositions, nature, and so on. For a specific size object, permittivity can be calculated by experimentally detected results via

$$
\varepsilon=\frac{C d}{S}
$$

Here $\varepsilon, C, d$, and $S$ denote the permittivity, capacitance, length, and cross-sectional area of the object, respectively. Often, relative permittivity $\left(\varepsilon_{r}\right)$ should be used to express electrical properties of materials. Relative permittivity of tissue is the ratio of permittivity of tissue $(\varepsilon)$ and the permittivity of vacuum $\left(\varepsilon_{0}\right)$; that is,

$$
\varepsilon_{r}=\frac{C d}{\varepsilon_{0} S} .
$$

When blood is concerned, it can be easily shaped for detecting the capacitance. But elastic properties of other tissues may be influenced by attached proteins on biological membranes [18], and the detecting capacitance may be affected by the detection voltage of the measuring equipment [19]. Therefore, it is necessary to ensure good contact between the test sample and the electrode. For the biological system, the dielectric property is generally expressed via the multiphase permittivity. Many classical formulae were put forward for calculating permittivity of the two-phase composition. Establishment of tissue permittivity model for biological system would help us to simplify its complex constitution.
A typical theory is Debye model, which is very famous and explains many compositions well and was put forward on the basis of empirical formula [20]. It is a widely used model for studying the dielectric properties of biological tissues in frequency domain. Binary mixtures' model is usually used for investigating effective permittivity of mixture system for its significance in understanding the intermolecular interactions. There are many permittivity models on binary mixtures without considering the frequency domain, for instance, the Bottcher-Bordewijk model [21], Maxwell-Garnett formula [22], Bruggeman formula [23], and Hanai formula [24]. Each theory can only be successfully applied to a certain type of composition. Among them, the Bottcher-Bordewijk model suggests very well feasibility to predict the permittivity of a mixture [21]. Here the model is as follows:

$$
\frac{3 \varepsilon_{1}}{2 \varepsilon+\varepsilon_{1}} \varphi_{1}+\frac{3 \varepsilon_{2}}{2 \varepsilon+\varepsilon_{2}} \varphi_{2}=1 .
$$

Here $\varepsilon_{i}$ denote the permittivity of the pure components and $\varphi_{i}$ denote the volume fraction of each of the components, so the equation can be modified as follows for two-phase composition:

$$
\frac{3 \varepsilon_{1}}{2 \varepsilon+\varepsilon_{1}} f+\frac{3 \varepsilon_{2}}{2 \varepsilon+\varepsilon_{2}}(1-f)=1 .
$$

Cell structure is of a random nature with some predictable average properties such as cell size and density. It is can be modeled by an aggregate of randomly distributed spherical shells in (4). This model can be used to describe the two constituent materials. When the two-phase system is concerned, there is another famous model put forward by Skipetrov [25]. Skipetrov equation is as follows:

$$
\varepsilon_{\mathrm{eff}}=\varepsilon_{1}\left(1+\frac{3 f\left(\varepsilon_{2}-\varepsilon_{1}\right)}{\varepsilon_{1}(2+f)+\varepsilon_{2}(1-f)}\right) .
$$

This model has been derived based on the assumption that $f$ here is much smaller than unity and that either correlation length or the particle diameter is well below the wavelength of electromagnetic waves used. The Skipetrov equation is original and more transparent than others and is assumed to give more correct results under tough situations. Skipetrov explored the effective permittivity of a random medium; the result showed that, for a two-component mixture, even volume fractions of one component $f \rightarrow 0$, the effective permittivity is also calculated and discussed in Skipetrov's work. Now, there is another famous model called Maxwell-Gannett model. Here we introduce the MaxwellGannett equation for the two-phase multisystem:

$$
\varepsilon_{\mathrm{eff}}=\varepsilon_{1}-3 f \varepsilon_{1} \frac{\varepsilon_{1}-\varepsilon_{2}}{\varepsilon_{2}+2 \varepsilon_{1}} .
$$

Here $\varepsilon_{\text {eff }}$ is the permittivity of the composite and $\varepsilon_{1}$ and $\varepsilon_{2}$ are permittivity of the doped phase and the matrix phase, respectively. So dielectric characteristics of tissues in human body are similar to the suspension system denoted by the Maxwell-Gannett conventional empirical models. Maxwell 
TABLE 1: $\varepsilon_{r}$ of several rat tissues.

\begin{tabular}{|c|c|c|c|c|}
\hline \multirow{2}{*}{ Tissue name } & \multicolumn{4}{|c|}{ Frequency $(\mathrm{Hz})$} \\
\hline & $10 \mathrm{k}$ & $100 \mathrm{k}$ & $1 \mathrm{M}$ & $10 \mathrm{M}$ \\
\hline Blood & $167370 \pm 28417$ & $28674 \pm 1262$ & $2459 \pm 224$ & $323 \pm 50$ \\
\hline Liver & $82306 \pm 20310$ & $18899 \pm 2675$ & $1833 \pm 255$ & $262 \pm 129$ \\
\hline Brain & $29693 \pm 5409$ & $4727 \pm 436$ & $1033 \pm 72$ & $245 \pm 69$ \\
\hline Fat & $8648 \pm 4311$ & $671 \pm 250$ & $147 \pm 28$ & $58 \pm 26$ \\
\hline
\end{tabular}

calculated permittivity of the composite media in the electrostatic field. With the in-depth study of the dielectric of the composite material, researchers are constantly looking for ways to model accurately the relationship between the various components of the composite material. When the dynamic electric field is further considered, Maxwell put out the permittivity model for complex biological tissue, which can be regarded as a small ball distributed in the continuum [22]. There are also other models, such as Bruggeman model [23], ideal mixture model [26], Peon-Iglesias model [27], and Kraszewski model [28]. All these models are been implicated to interpret the effective permittivity in two-phase composite material [29].

When biological tissue is concerned, uneven distribution of blood cells in tissues makes its representative dielectric properties [30]. Additionally, biological membranes are charged the dielectric properties, and in the vicinity of the membrane they are strongly influenced by orientational ordering of water dipoles which contributes to strong decrease of relative permittivity in the vicinity of the cell membranes and also partially within the membranes in their surface region. Therefore, accurately, the dielectric properties cannot be calculated from traditional models. In the following paper, we will discuss the applicability of these models for biological tissues from the perspective of experiment and theory, especially the deviation of these models from the experimental results.

\section{Experimental Results}

Weight of male Wistar rats is $200 \pm 10 \mathrm{~g}$. Intraperitoneal injection of sodium pentobarbital is conducted with dose of $40 \mathrm{mg} / \mathrm{kg}$. The chests of anesthetized rats were cut after being fixed on the disinfection board; $3-5 \mathrm{~mL}$ blood was acupunctured from left apex of heart; and then the blood was put into the plexiglass tank (size: $6 \mathrm{~mm} \times 6 \mathrm{~mm} \times 40 \mathrm{~mm}$ ); all results are measured by HP4275A Multi-Frequency LCR Meter from $10 \mathrm{kHz}$ to $10 \mathrm{MHz}$. Than brain, liver, and fat were measured in a similar way. Permittivity can be calculated via (1). Often, relative permittivity should be used to express electrical properties of materials, and relative permittivity of tissue is the ratio of permittivity of tissue and the permittivity of vacuum [31]; that is, $\varepsilon_{r}=\varepsilon / \varepsilon_{0}$. The relative permittivity of rat tissues is shown in Table 1 . Here, $\varepsilon_{r}$ of all tissues decline with the frequency; the main reason for the phenomena is that there is a period of time (relaxation time); different relaxation times characterize special responses of tissues to external electric fields.
Blood is one of the most important components in body, and its biological function is mainly to complete the transport of oxygen and nutrients. Dielectric properties of blood can reflect the composition of blood tissue, which can be used for composition analysis of intracellular fluid [32]. Water molecules in blood tissue will be reoriented under external electric field. The polarization process of water molecules in blood tissue under external field is not instantly stable when the external field changes.

Liver as a large organ plays an important role in metabolism and regulation of glycogen storage, which processes plenty of blood. From Table $1, \varepsilon_{r}$ in rat liver from fell from $82306(10 \mathrm{kHz})$ to $262(10 \mathrm{MHz})$, where $\varepsilon_{r 10 \mathrm{kHz}} / \varepsilon_{r 10 \mathrm{MHz}}=314.1$, which indicates that its large water content is mainly because of its large blood content. Electrical characteristics of the brain have a wide range of applications and take its basis for EEG; permittivity and conductivity can be applied to the position detection of brain function for inverse problems. The relative permittivity of the brain tissue is relatively low, which is related to the microstructure of the brain tissue itself and indicates that the content of polar molecules is relatively small. Here, $\varepsilon_{r 10 \mathrm{kHz}} / \varepsilon_{r 10 \mathrm{MHz}}=121.2$ indicate that its water content is lower than the blood [33]. Fat has been considered to have poor conductivity. Here, $\varepsilon_{r 10 \mathrm{kHz}} / \varepsilon_{r 10 \mathrm{MHz}}=149.7$ indicating the small content of water molecular. Experimental results here meet the actual composition of adipose tissue composition. If the tested tissue departed from body too long, water losing would affect the test results.

In addition, (2) is the basic method we adopted to calculate $\varepsilon_{r}$; however, calculated results may have a deviation from actually value due to the shape of parallel plate capacitor which may have a certain edge effect. But for the analysis of the $\varepsilon_{r 10 \mathrm{kHz}} / \varepsilon_{r 10 \mathrm{MHz}}$ value and the applicability of effective permittivity model, it would not bring any adverse effects.

\section{Comparison of Theory and Experiment}

Many researchers have carried out theoretical and experimental studies on the dielectric properties of compositions [34]. Because the effective permittivity is related to the structure and the properties of the composite materials, there are some inherent flaws in describing the physical properties of the additives in the medium [35]. Most of the theoretical researches are concerned with the volumetric fraction which cannot be determined accurately [36]. Most of the existing research work has not taken into account the influence of the frequency. As a result, these classical theories are subject to a 
number of constraints. Each theory can only be successfully applied to a certain type of composite material, but none of them can explain all types of composite materials. Here we use the above experimental data and theoretical model to analyze the value of the volume fraction parameter.

In order to clearly express the volume fraction, we transform (4), (5), and (6). Here, we concern volume fraction $f$; from the Bottcher-Bordewijk model (see (4)), we get

$$
f=\frac{2 \varepsilon^{2}+\varepsilon_{1} \varepsilon-2 \varepsilon_{2} \varepsilon-\varepsilon_{1} \varepsilon_{2}}{3 \varepsilon_{1} \varepsilon-3 \varepsilon_{2} \varepsilon} .
$$

Bottcher-Bordewijk model is a relatively simple model and does not specify the base phase and the doping phase, but it describes the composite system of volume fraction via weight ratio. The Skipetrov model described in (5) specifies the applicable conditions, which take into account a dilute suspension where dielectric constant of spherical particles and matrix medium are $\varepsilon_{2}$ and $\varepsilon_{1}$, respectively. However, for the liver and brain tissue, the blood-fat composition model is not in extreme circumstances. For convenience, here $\varepsilon_{1}$ denotes the permittivity in blood, and $\varepsilon_{2}$ is the permittivity in fat. So, from the Skipetrov equation, we obtain the following expression:

$$
f=\frac{\varepsilon_{1} \varepsilon_{2}-2 \varepsilon \varepsilon_{1}-\varepsilon \varepsilon_{2}+2 \varepsilon_{1}^{2}}{\varepsilon \varepsilon_{1}-\varepsilon \varepsilon_{2}-2 \varepsilon_{1} \varepsilon_{2}+2 \varepsilon_{1}^{2}} .
$$

Maxwell-Gannett theory is actually a mean field theory. It assumes that an external electric field is applied to the composite, and then the electric field intensity and the electric displacement vector are calculated by the electric field theory. The electric field intensity and the electric displacement vector are then summed by the volume, so quotient of the electric displacement vector divided by the sum of the electric field strength is the permittivity of the composite material. From the Maxwell-Gannett equation (see (6)), we get

$$
f=\frac{\left(2 \varepsilon_{1}+\varepsilon_{2}\right)\left(\varepsilon_{1}-\varepsilon\right)}{3 \varepsilon_{1}\left(\varepsilon_{1}-\varepsilon_{2}\right)} .
$$

The data in Table 1 are substituted into (7), (8), and (9), and then we obtain the results as shown in Figures 2 and 3.

From microlevel, dipole moment of a water molecule in tissue differs from that of an isolated one [37]. This is mainly because water molecule in tissue is further polarized by effect by its nearby neighbors and the biomolecules [38, 39]. The blood volume fraction is unstable as the frequency changes as in Figures 2 and 3. There is no theoretical formula to express the composite dielectric properties of liver tissue and brain tissue with the same $f$ value at different frequencies. It can be predicted that the effective permittivity of the material will be underestimated by using the simply tandem model (see (4)). The serial-parallel models (see (5) and (6)) will overestimate the predicted value. The main reason for the above phenomenon is that the tissues also have the characteristics of dielectric dispersion. Because of the effect from the proteins in cell membrane, the fatty acids, other macromolecules, the intracellular, liver, and brain can be mixed as a suspension

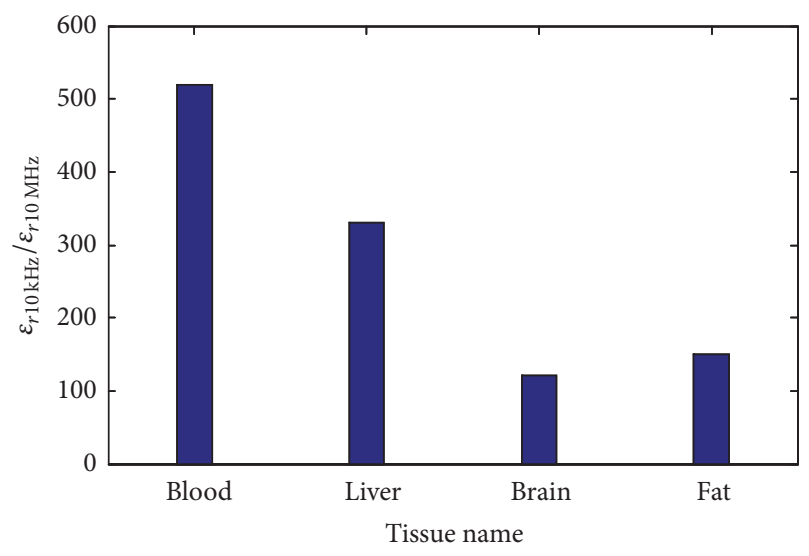

FIGURE 1: $\varepsilon_{r 10 \mathrm{kHz}} / \varepsilon_{r 10 \mathrm{MHz}}$ value of different tissues.

system, not only the blood and fat. On the other hand, the interface polarization occurs on blood cell during charging and discharging when the external electric field exists. When we calculate the volume fraction, the interaction between different kinds of cell is not considered here. Considering the mechanism of the dielectric fluid within the tissues, the cell membrane and intracellular fluid could be regarded further as whole unit. From this perspective, the previous model we used is suitable for tissues. From the Figures 2 and 3, volume fraction at different frequency denotes that the relaxation of dielectric spectroscopy is closely related to the typical biological tissues, so the internal information of tissues can not be explained by these models.

The Maxwell-Gannett theory is suitable for the case where one particle is surrounded by another substrate. However, the theory can not explain the phenomenon that is dielectric dispersion in composite system, and it also can not judge which material is the matrix. Prodan et al. studied the polarization properties of the cell membrane theoretically via calculating the effect of electric field, the results specifically show that the permittivity of the cell membrane decreased with the increasing frequency [16], and the permittivity of the cell membrane is close to zero when the membrane is in high frequency $\left(>10^{5} \mathrm{~Hz}\right)$.

Impact of biological activity, such as connection and communication between cells and cells, should be considered in analyzing characteristics of dielectric dispersion in biological tissue, especially in analyzing $\alpha$-dispersion zone. Generally, membrane potential controls the height of the $\alpha$-dispersion, and the mobility of the surface charge accumulated on the cell membrane controls its length. In the analysis of $\beta$ dispersion area it should consider the organelle, cytoplasm, and components of the biotissues and the water content of them in the analysis of $\gamma$ dispersion. From this perspective, in Table 1 and Figure 1, although the value of $\varepsilon_{r 10 \mathrm{kHz}} / \varepsilon_{r 10 \mathrm{MHz}}$ reaches several hundreds, $\varepsilon_{r}$ is still 200 or so. On the other hand, Huclova et al. established a reliable model of cell organization by taking ion channels, proteins, and organelles and others into account. The results show that dielectric dispersion of biological tissue in megahertz range may be 


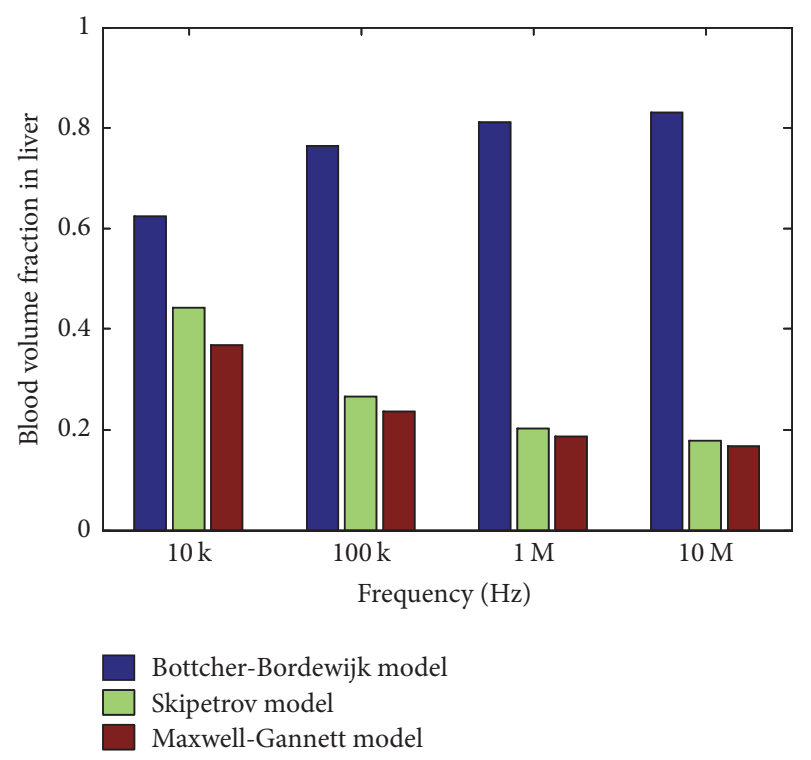

FIGURE 2: Blood volume fraction in liver under three typical models.

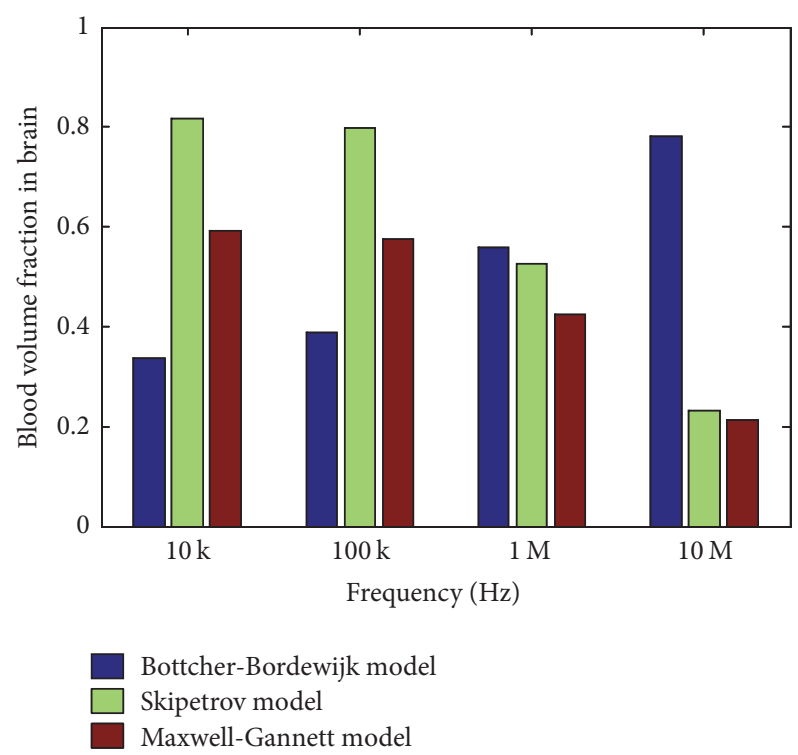

FIGURE 3: Blood volume fraction in brain under three typical models.

due to interface polarization of the cell membrane; we find in Figures 2 and 3 that the volume fraction of blood is become lower when the frequency increases according to Skipetrov and Maxwell-Gannett models. So, from the perspective of dielectric dispersion in biological tissues, Skipetrov and Maxwell-Gannett models in Figures 2 and 3 could meet the findings by Huclova et al. [40]. For a given cell density and geometry of the biological tissue, its low frequency dielectric response is related to the size of the cell membrane potential. There are some composite materials models for predicting the dielectric properties of biotissues; all of them cannot interpret the dispersion area except Debye model. So here we compare several static common model of their applicability for interpreting the dielectric properties of the biological system in the frequency range.

\section{Conclusions}

From the perspective of electromagnetism, organisms are composed of a large number of cells with different electromagnetic properties. Biological characteristics of tissue under different electromagnetic waves are the corresponding key issue for studying the biological effects of electromagnetic fields. This paper studied the effective permittivity parameter of blood, liver, brain, and fat from the theoretical and experimental point of view. Mechanism is analyzed on effective permittivity in tissues based on its typical composition via several typical models in this paper. Volume fraction of blood in liver and brain is analyzed based on the BottcherBordewijk model, the Skipetrov equation, and the MaxwellGannett theory theoretically at different frequencies. The results show that the volume fraction of blood is not stable from $10 \mathrm{kHz}$ to $10 \mathrm{MHz}$, so these models all have some limitations in interpreting the effective permittivity characteristic in biotissues under the action of dynamic voltage. This is mainly because of the changes of dielectric properties of biological tissues with frequency which is very different. It is necessary to establish distribution model suitable for the dielectric properties of different constituents in frequency domain, especially the theoretical model which can reflect the characteristics of the dielectric dispersion. It is very meaningful to use the composite media model to explain the dielectric properties of the biological system for their importance in the biomedical imaging, biomimetic material design, and physical therapy of the disease.

\section{Conflicts of Interest}

The authors declare that they have no conflicts of interest.

\section{Acknowledgments}

This research work was supported by schoolmaster fund of Xian Technological University (no. XAGDXJJ14011).

\section{References}

[1] J. Leroy, C. Dalmay, A. Landoulsi et al., "Microfluidic biosensors for microwave dielectric spectroscopy," Sensors and Actuators, A: Physical, vol. 229, pp. 172-181, 2015.

[2] F. Farsaci, E. Tellone, M. Cavallaro, A. Russo, and S. Ficarra, "Low frequency dielectric characteristics of human blood: a non-equilibrium thermodynamic approach," Journal of Molecular Liquids, vol. 188, pp. 113-119, 2013.

[3] G. Li and X.-F. Pang, "Effects of electromagnetic field exposure on electromagnetic properties of biological tissues," Progress in Biochemistry and Biophysics, vol. 38, no. 7, pp. 604-610, 2011.

[4] E. Marzec and W. Warchol, "Dielectric properties of a proteinwater system in selected animal tissues," Bioelectrochemistry, vol. 65, no. 2, pp. 89-94, 2005. 
[5] E. Marzec, P. Sosnowski, J. Olszewski et al., "Dielectric relaxation of normothermic and hypothermic rat corneas," Bioelectrochemistry, vol. 101, pp. 132-137, 2015.

[6] T. Dai and A. Adler, "In vivo blood characterization from bioimpedance spectroscopy of blood pooling," IEEE Transactions on Instrumentation and Measurement, vol. 58, no. 11, pp. 3831-3838, 2009.

[7] Y. Otaki, Y. Ebana, S. Yoshikawa, and M. Isobe, "Dielectric permittivity change detects the process of blood coagulation: comparative study of dielectric coagulometry with rotational thromboelastometry," Thrombosis Research, vol. 145, pp. 3-11, 2016.

[8] Y. Llave, K. Mori, D. Kambayashi, M. Fukuoka, and N. Sakai, "Dielectric properties and model food application of tylose water pastes during microwave thawing and heating," Journal of Food Engineering, 2015.

[9] N. Simicevic and D. T. Haynie, "FDTD simulation of exposure of biological material to electromagnetic nanopulses," Physics in Medicine and Biology, vol. 50, no. 2, pp. 347-360, 2005.

[10] N. Jebbor and S. Bri, "Effective permittivity of periodic composite materials: numerical modeling by the finite element method," Journal of Electrostatics, vol. 70, no. 4, pp. 393-399, 2012.

[11] M. Piralaee, A. Asgari, and V. Siahpoush, "Modeling and optimizing the performance of plasmonic solar cells using effective medium theory," Physics Letters A, vol. 381, no. 5, pp. 489-493, 2017.

[12] M. N. Rylander, Y. Feng, Y. Zhang et al., "Optimizing heat shock protein expression induced by prostate cancer laser therapy through predictive computational models," Journal of Biomedical Optics, vol. 11, no. 4, p. 041113, 2006.

[13] A. Prasad and K. Prasad, "Effective permittivity of random composite media: a comparative study," Physica B: Condensed Matter, vol. 396, no. 1-2, pp. 132-137, 2007.

[14] A. A. Amooey, "Improved mixing rules for description of the permittivity of mixtures," Journal of Molecular Liquids, vol. 180, pp. 31-33, 2013.

[15] T. I. Zohdi, F. A. Kuypers, and W. C. Lee, "Estimation of red blood cell volume fraction from overall permittivity measurements," International Journal of Engineering Science, vol. 48, no. 11, pp. 1681-1691, 2010.

[16] E. Prodan, C. Prodan, and J. H. Miller Jr., "The dielectric response of spherical live cells in suspension: An analytic solution," Biophysical Journal, vol. 95, no. 9, pp. 4174-4182, 2008.

[17] J. Peón-Fernández, J. Martín-Herrero, N. Banerji, and T. P. Iglesias, "Numerical study of effective permittivity in composite systems," Applied Surface Science, vol. 226, no. 1-3, pp. 78-82, 2004.

[18] A. Iglič, T. Slivnik, and V. Kralj-Iglič, "Elastic properties of biological membranes influenced by attached proteins," Journal of Biomechanics, vol. 40, no. 11, pp. 2492-2500, 2007.

[19] A. Velikonja, V. Kralj-Iglic, and A. Iglic, "On asymmetric shape of electric double layer capacitance curve," International Journal of Electrochemical Science, vol. 10, no. 1, p. 1.

[20] P. Zakharov, F. Dewarrat, A. Caduff, and M. S. Talary, “The effect of blood content on the optical and dielectric skin properties," Physiological Measurement, vol. 32, no. 1, pp. 131-149, 2011.

[21] T. H. Tjia, P. Bordewijk, and C. J. F. Böttcher, "On the notion of dielectric friction in the theory of dielectric relaxation," Advances in Molecular Relaxation Processes, vol. 6, no. 1, pp. 1928, 1974.
[22] J. C. Maxwell-Garnett, "Colours in metal glasses and metal films," Philosophical Transactions of the Royal Society A Mathematical Physical \& Engineering Sciences, vol. 203, no. 359-371, pp. 385-420, 1904.

[23] M. Bruggerman, W. Kalkner, A. Campus, and A. Smedberg, "Electrochemical effects at the conductor/dielectric interface-a description of the mechanism," IEEE International Conference on Solid Dielectrics, vol. 1, pp. 383-386, 2004.

[24] T. Hanai, "A remark on theory of dielectric dispersion due to interfacial polarization," Colloid Polymer Science, vol. 175, no. 1, pp. 61-62, 1961.

[25] S. E. Skipetrov, "Effective dielectric function of a random medium," Physical Review B - Condensed Matter and Materials Physics, vol. 60, no. 18, pp. 12705-12709, 1999.

[26] A. H. Buep, "Dielectric properties of liquid systems: the ideal complex permittivity in liquid mixtures," Turkish Journal of Physics, vol. 33, no. 5, pp. 243-247, 2009.

[27] J. F. Peón-Fernández and T. P. Iglesias, "On the permittivity of mixtures: a low-fluctuation approach," Journal of Electrostatics, vol. 32, no. 2, pp. 113-122, 1994.

[28] A. Kraszewski, M. A. Stuchly, S. S. Stuchly, and A. M. Smith, "In vivo and in vitro dielectric properties of animal tissues at radio frequencies," Bioelectromagnetics, vol.3, no. 4, pp. 421-432, 1982.

[29] T. Sandu, D. Vrinceanu, and E. Gheorghiu, "Linear dielectric response of clustered living cells," Physical Review E - Statistical, Nonlinear, and Soft Matter Physics, vol. 81, no. 2, Article ID 021913, 2010.

[30] A. Di Biasio and C. Cametti, "Polarizability of spherical biological cells in the presence of localized surface charge distributions at the membrane interfaces," Physical Review EStatistical, Nonlinear, and Soft Matter Physics, vol. 82, no. 2, Article ID 021917, 2010.

[31] W. J. K. Raymond, C. K. Chakrabarty, G. C. Hock, and A. B. Ghani, "Complex permittivity measurement using capacitance method from $300 \mathrm{kHz}$ to $50 \mathrm{MHz}$," Measurement, vol. 46, no. 10, pp. 3796-3801, 2013.

[32] H. F. Cook, "A comparison of the dielectric behaviour of pure water and human blood at microwave frequencies," British Journal of Applied Physics, vol. 3, no. 8, article 302, pp. 249-255, 1952.

[33] Y. Polevaya, I. Ermolina, M. Schlesinger, B.-Z. Ginzburg, and Y. Feldman, "Time domain dielectric spectroscopy study of human cells II. Normal and malignant white blood cells," Biochimica et Biophysica Acta-Biomembranes, vol. 1419, no. 2, pp. 257-271, 1999.

[34] H. He, M. Dyck, Y. Zhao et al., "Evaluation of five composite dielectric mixing models for understanding relationships between effective permittivity and unfrozen water content," Cold Regions Science and Technology, vol. 130, pp. 33-42, 2016.

[35] Y. G. Smirnov, M. Y. Medvedik, and E. E. Grishina, "Determination of the effective permittivity of a body in a waveguide from the reflection coefficient," Journal of Communications Technology and Electronics, vol. 59, no. 2, pp. 145-149, 2014.

[36] S. Moiseev and V. Ostatochnikov, "Influence of the sizedependent permittivity of metal inclusions on the optical characteristics of a one-dimensional photonic crystal with a nanocomposite defect," Optical and Quantum Electronics, vol. 47, no. 9, pp. 3193-3200, 2015.

[37] E. Gongadze and A. Iglič, "Decrease of permittivity of an electrolyte solution near a charged surface due to saturation and excluded volume effects," Bioelectrochemistry, vol. 87, pp. 199203, 2012. 
[38] S. M. A. Jiménez and R. M. McMeeking, "A constitutive law for dielectric elastomers subject to high levels of stretch during combined electrostatic and mechanical loading: Elastomer stiffening and deformation dependent dielectric permittivity," International Journal of Non-Linear Mechanics, vol. 87, pp. 125136, 2016.

[39] X. F. Pang, S. Chen, X. Wang, and L. Zhong, "Influences of electromagnetic energy on bio-energy transport through protein molecules in living systems and its experimental evidence," International Journal of Molecular Sciences, vol. 17, no. 8, article 1130, 2016.

[40] S. Huclova, D. Erni, and J. Fröhlich, "Modelling effective dielectric properties of materials containing diverse types of biological cells," Journal of Physics D: Applied Physics, vol. 43, no. 36, Article ID 365405, 2010. 


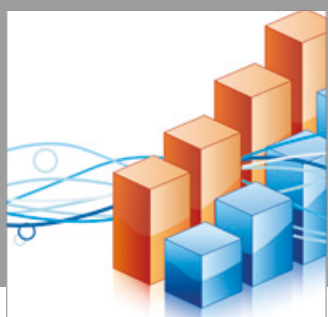

Advances in

Operations Research

vatersals

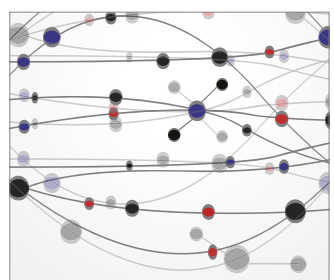

\section{The Scientific} World Journal
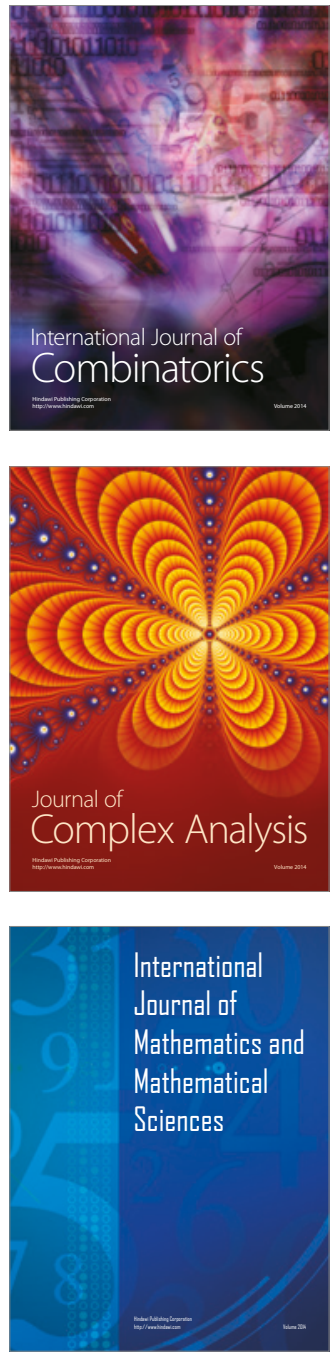
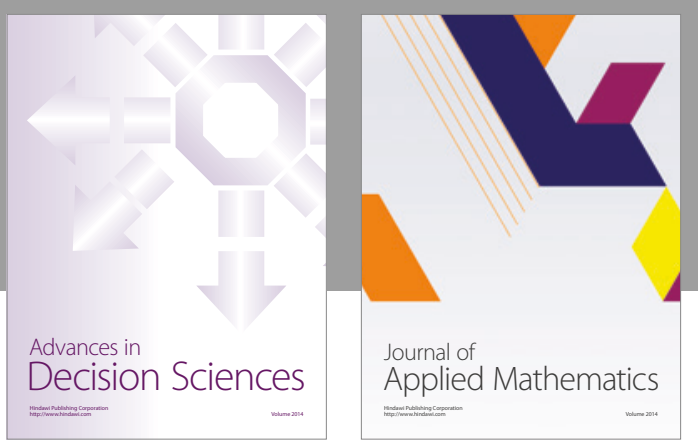

Algebra

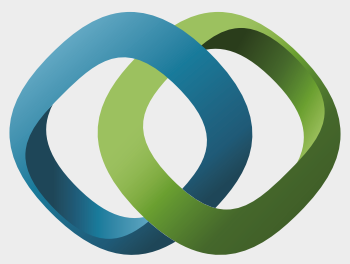

\section{Hindawi}

Submit your manuscripts at

https://www.hindawi.com
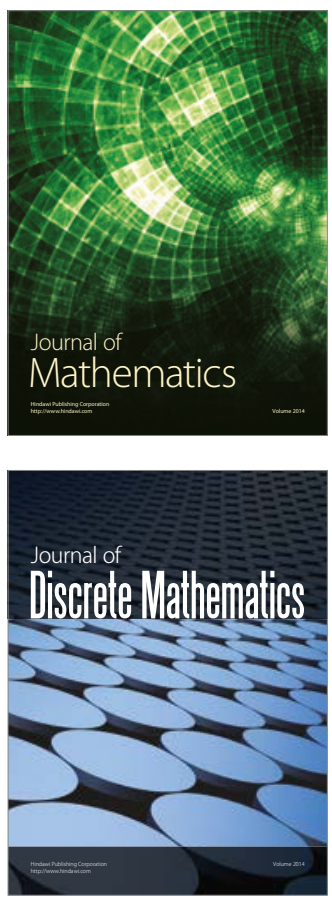

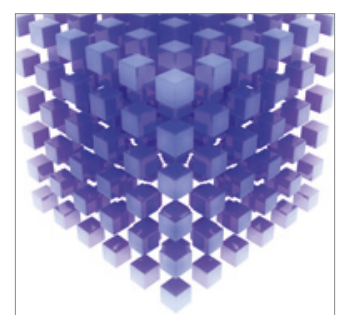

Mathematical Problems in Engineering
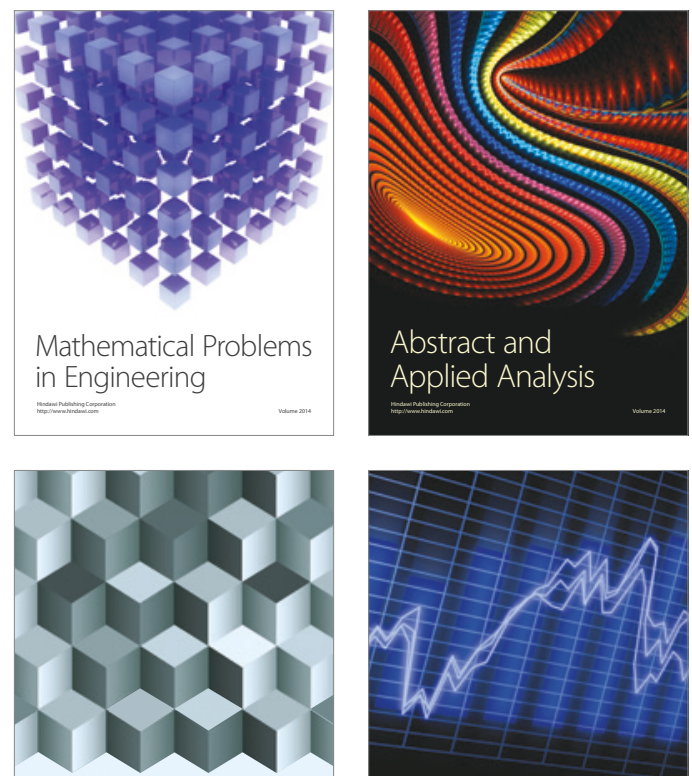

Journal of

Function Spaces

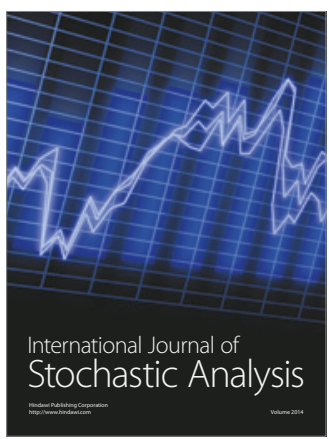

Probability and Statistics
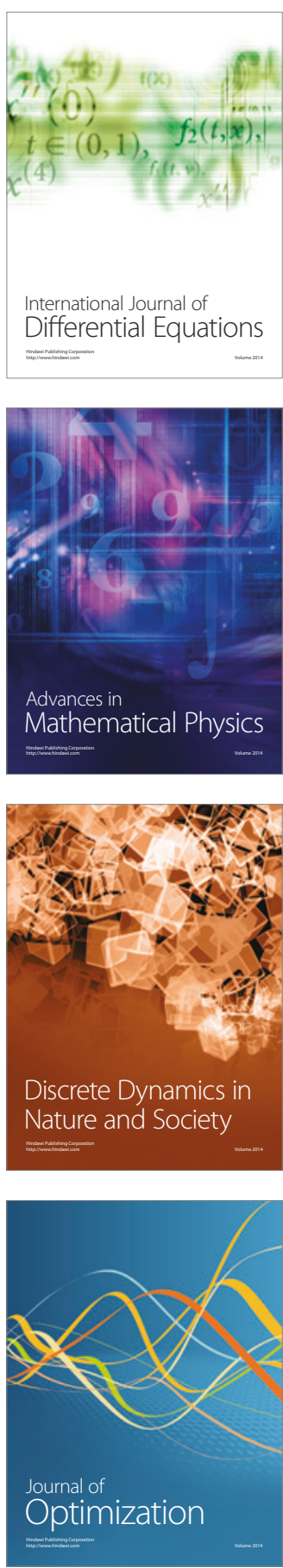